\title{
Integrated Management of Collar Rot of Chilli Caused by Sclerotium rolfsii Sacc
}

\author{
A. T. Daunde ${ }^{\text {* }}$, K. T. Apet ${ }^{2}$, K. D. Navgire ${ }^{2}$ and V.S. Khandare ${ }^{1}$ \\ ${ }^{1}$ AICRP (Vegetable Crops), ${ }^{2}$ Department of Plant Pathology, Vasantrao Naik Marathwada \\ Krishi Vidyapeeth, Parbhani (MS) 431 402, India \\ *Corresponding author
}

\section{A B S T R A C T}

\begin{tabular}{l} 
K e y w o r d s \\
Sclerotium rolfsii, \\
Chilli, Integrated \\
management, \\
Carbendazim + \\
Mancozeb, \\
Trichoderma viride, \\
Neem seed cake \\
\hline Article Info \\
\hline $\begin{array}{l}\text { Accepted: } \\
18 \text { May } 2020 \\
\text { Available Online: } \\
\text { 10 June } 2020\end{array}$ \\
\hline
\end{tabular}

\section{Introduction}

Chilli (Capsicum annuum L.) is one of the most important constituents of the foods of tropical and subtropical countries and the fourth major crop cultivated worldwide. Their characteristics such as pungency, colour, aroma, and flavour are desirable in a variety of culinary dishes around the world, which make them widely appreciated. Chilli is susceptible to many diseases, some of them are widespread and some are localized.
Collar rot (Sclerotium rolfsii Sacc.) of chilli (Capsicum annuum L.) is one of the devastating soil-borne diseases of fungal origin, due to which $16-80 \%$ yield loss is recorded annually according to the severity of the disease. An integrated disease management approach was developed for the management of collar rot of chilli using fungicide, biocontrol agents and an organic amendment. The results of the experiments from the two years pooled data and cost-benefit ratio indicated that chilli seedling dip treatment with Carbendazim $12 \%$ + Mancozeb $63 \%$ (75 WP) @ $2.5 \mathrm{~g} / 1+$ Trichoderma viride @ $10 \mathrm{~g} / \mathrm{l}+$ soil application with Neem seed cake @ $5 \mathrm{q} / \mathrm{ha}$ significantly reduced the collar rot disease incidence $(8.33 \%)$ with higher $\mathrm{B}: \mathrm{C}$ ratio $(3.73: 1)$. The reduction in disease incidence over control in this treatment was $77.06 \%$. Consequently, the green fruit yield in chilli was 128.11 $\mathrm{q} / \mathrm{ha}$, recording a percent increase of $68.72 \%$ over control. The integrated management of chilli collar rot by using fungicide, biocontrol agents and an organic amendment is safe and ecologically sound and appears to be a healthy approach to the disease management. 
Management of collar rot/root rot diseases is difficult because of soil borne nature and the chemical methods are very expensive and will not be that good effect against the pathogen and also have a wide host range. Because of unsatisfactory control of soil borne pathogen by chemicals, considerable attention has been given on the other non-chemical means of plant disease control. This can be effectively managed by the integration of biological and conventional methods, which include the use of eco-friendly biocontrol agents.

The application of biocontrol agents to the soil is an alternative to suppress soil borne plant pathogens through parasitism, production of antagonistic chemicals, competition for the host and nutrients, and induction of resistance in plants against disease-causing pathogens (Shafique, 2016).Biological control of soil borne pathogens offersan environmentally safe, durable and cost-effective alternative to chemicals (Sharma, 2011). Many species of fungi and bacteria are reported to be effective as bio-control agents against soil borne plant pathogens (Papavizas, 1985; Baker, 1987).

Integration of biological seed protectant with fungicidal, insecticidal treatment involves, the reduced amounts of pesticides can stress and weaken the pathogen and render its propagules more susceptible to subsequent attack by the antagonist and provides better control of seeds and seedlings diseases than either used separately (Lorito et al., 1996; Mahato et al., 2017). Hence, the integrated approach plays an important role in the effective management of collar rot of chilli.

Integrated Pest Management strategy is comparatively safe, environment friendly and durable. Keeping these in view an experiment was undertaken to evaluate the effectiveness of integrated disease management strategies involved of fungicide, bio-control agent and organic amendment to manage collar rot $(S$. rolfsii) disease of chilli.

\section{Materials and Methods}

The experiment was conducted at Horticulture Research Scheme (Vegetable), Vasantrao Naik Marathwada Krishi Vidyapeeth, Parbhani, Maharashtra, India during the year 2016-17 to 2017-18 in Kharif seasons using chilli cv. Pusa Jwala for the management of collar rot of chilli caused by Sclerotium rolfsii Sacc. The soil of the experimental field was black cotton soil. The experiment was laid out in a randomized block design with three replications. The plot size was $2.4 \times 3.15 \mathrm{~m}$. Thirty-five days old seedlings raised in the nursery were transplanted at a distance of 60 $\mathrm{x} 45 \mathrm{~cm}$ spacing. Efficacies of twelve treatments as shown in Table 1 were compared with untreated control. The seedlings of chilli were dipped in the suspension of bioagents and fungicides for 15 minutes just before transplanting in the field. All recommended horticultural practices were followed. Observations on the incidence of collar rot disease were recorded and statistically analysed. Per cent disease incidence was calculated by using the following formula.

Disease incidence $(\%)=\frac{\text { Number of diseased plants }}{\text { Total number of plants observed }} \times 100$

\section{Results and Discussion}

\section{Effect of various treatments on the disease incidence of collar rot of chilli}

The data on disease incidence presented in Table 2 showed that all the treatments reduced the disease incidence significantly as compared to untreated control. It is revealed from the data that there was significant difference in percent disease incidence during 2016-17 and 2017-18 and pooled also. 
In pooled data, the minimum disease incidence was noticed in treatment $\mathrm{T}_{9}$ (Carboxin $37.5 \%$ + Thiram $37.5 \%$ (75 WP) + Trichoderma harzianum + Neem seed cake) $\left(4.17 \%\right.$ ) followed by $\mathrm{T}_{10}$ (Carbendazim $12 \%$ + Mancozeb $63 \%(75 \mathrm{WP})+T$. viride + Neem seed cake) $(8.33 \%), \mathrm{T}_{7}$ ( Carbendazim $50 \mathrm{WP}+$ Trichoderma harzianum + Neem seed cake) $(10.71 \%), \mathrm{T}_{8}$ (Carbendazim 50 $\mathrm{WP}+T$. viride + Neem seed cake) $(11.91 \%)$, $\mathrm{T}_{3}$ (Carboxin $37.5 \%+$ Thiram $37.5 \%$ (75 WP) (13.10\%), $\mathrm{T}_{11}$ (Bioagents consortia) $(16.67 \%), \quad \mathrm{T}_{2}$ (Carbendazim $12 \%+$ Mancozeb $63 \%$ (75 WP) (20.84\%), $\mathrm{T}_{2}$ (Carbendazim 50 WP (22.62 \%), $\mathrm{T}_{1}$ (Captan $50 \mathrm{WP})(24.41 \%), \mathrm{T}_{5}$ (T. harzianum) $(25.60$ $\%)$ and $\mathrm{T}_{6}($ T. viride $)(27.98 \%)$.

\section{Effect of various treatments on the yield of} collar rot of chilli

Effect of different treatments on chilli yield was found significant during all the years andpooled also (Table 3). The data on chili green fruit yield showed that all the treatments recorded significantly higher yield as compared to untreated control. In pooled data, significantly highest yield of 148.15 $\mathrm{q} / \mathrm{ha}$ was recorded by treatment $\mathrm{T}_{9}$ (Carboxin $37.5 \%$ + Thiram $37.5 \%$ (75 WP) + Trichoderma harzianum + Neem seed cake). This was followed by the treatment viz., $\mathrm{T}_{10}$ (Carbendazim $12 \%$ + Mancozeb $63 \%$ (75 $\mathrm{WP})+T$. viride + Neem seed cake) (128.11 $\mathrm{q} / \mathrm{ha}), \mathrm{T}_{8}$ (Carbendazim $50 \mathrm{WP}+$ T. viride + Neem seed cake) (126.46 q/ha), $\mathrm{T}_{7}$ (Carbendazim 50 WP+ Trichoderma harzianum + Neem seed cake) (125.22 q/ha), $\mathrm{T}_{2}$ (Carbendazim $12 \%$ + Mancozeb 63 $\% \quad(75 \mathrm{WP})(118.12 \mathrm{q} / \mathrm{ha}), \mathrm{T}_{11}$ (Bioagents consortia) (115.82 q/ha), $\mathrm{T}_{3}$ (Carboxin $37.5 \%$ + Thiram $37.5 \%$ (75 WP) (115.00 q/ha), $\mathrm{T}_{2}$ (Carbendazim 50 WP(111.87 q/ha), $\mathrm{T}_{1}$ (Captan 50 WP) $\left(107.35\right.$ q/ha), $\mathrm{T}_{5} \quad(T$. harzianum) (97.35 q/ha) and $\mathrm{T}_{6}(T$. viride $)$ (95.17 q/ha).
Economics of integrated management practices on collar rot of chilli

The economic evaluation of the experiments was done to evaluate the best treatment in terms of monitory units. The economics of benefit: cost ratio was worked out and presented in Table 4 revealed that, treatment $\mathrm{T}_{10}$ (Carbendazim $12 \%+$ Mancozeb $63 \%$ (75 $\mathrm{WP})+T$. viride + Neem seed cake) obtained highest gross income (Rs. 4,44,444/ha), net profit (Rs.3,50,573/ha) and B:C ratio (3.73:1).

The second best treatment $\mathrm{T}_{9}$ (Carboxin 37.5 $\%$ + Thiram $37.5 \%$ (75 WP) + Trichoderma harzianum + Neem seed cake) which was superior concerning yield (148.15 q/ha) and recorded less B:C ratio (3.15:1), mainly because of the increased cost of chemicals.

Rest of the treatments found economical with better B:C ratio were viz., $\mathrm{T}_{7}$ ( Carbendazim $50 \mathrm{WP}+$ Trichoderma harzianum + Neem seed cake), $\mathrm{T}_{8}$ (Carbendazim $50 \mathrm{WP}+T$. viride + Neem seed cake), $\mathrm{T}_{3}$ (Carboxin 37.5 $\%$ + Thiram $37.5 \%$ (75 WP)), $\mathrm{T}_{11}$ (Bioagents consortia), $\mathrm{T}_{2}$ (Carbendazim $12 \%+$ Mancozeb $63 \%$ (75 WP), $\mathrm{T}_{2}$ (Carbendazim $50 \mathrm{WP}, \mathrm{T}_{1} \quad$ (Captan $\left.\left.50 \mathrm{WP}\right)\right), \mathrm{T}_{5} \quad(T$. harzianum) and $\mathrm{T}_{6}$ (T. viride).

The results of the experiments from the two years data clearly indicated that chilli seedling dip treatment with Carbendazim $12 \%+$ Mancozeb $63 \%$ (75 WP) @ $2.5 \mathrm{~g} / \mathrm{l}+$ Trichoderma viride @ $10 \mathrm{~g} / \mathrm{l}+$ soil application with Neem seed cake@ 5 q/ha significantly reduced the collar rot disease incidence $(8.33 \%)$.

The reduction in disease incidence over control in this treatment was $77.06 \%$. Consequently, the fruit yield in chilli was $128.11 \mathrm{q} / \mathrm{ha}$, recording a per cent increase of $68.72 \%$ over control. The possible reason for such finding may be that Carbendazim + 
Mancozeb would have affected the spore germination and mycelial development, antagonistic effect of Trichoderma viride and neem seed cake plays an important role in reducing the incidence of soil borne diseases which may have resulted in the inhibition of disease producing activity of pathogen in the plant and induced resistance in the plant. Kowsari, 2014 stated that Trichoderma species are known to produce large quantities of fungi-toxic metabolites. They are the active mycoparasites which have been used as effective biocontrol agents against foliar and soil borne disease. This resulted in better overall growth and good health of chilli plants. This may be the reason for minimum disease incidence and maximum yield as compared to other treatments.

Similar views were put forth by several other workers (Dutta and Das, 2002; Vanitha and Suresh, 2002; Bhoraniya et al., 2003; Gogoi et al., 2004; Islam and Bhuiyan, 2006; Okereke et al., 2007; Banyal et al., 2008; Jadon, 2009; Begum et al., 2011; Madhavi and Bhattiprolu, 2011; Sultana et al., 2012; Bhattacharjee et al., 2015; Suryawanshi et al., 2015; Kuldhar and Suryawanshi, 2017).

Table.1 Details of various treatments used for the management of chilli collar rot

\begin{tabular}{|c|l|c|}
\hline $\begin{array}{c}\text { Treatment } \\
\text { No. }\end{array}$ & \multicolumn{1}{|c|}{ Treatments } & $\begin{array}{c}\text { Doses } \\
\text { (g/l of water or q/ha } \\
\text { of soil) }\end{array}$ \\
\hline $\mathbf{T}_{\mathbf{1}}$ & Captan 50 WP & $3.0 \mathrm{~g}$ \\
\hline $\mathbf{T}_{\mathbf{2}}$ & Carbendazim 50 WP & $1.0 \mathrm{~g}$ \\
\hline $\mathbf{T}_{\mathbf{3}}$ & Carboxin 37.5\% + Thiram 37.5\% (75 WP) & $3.0 \mathrm{~g}$ \\
\hline $\mathbf{T}_{\mathbf{4}}$ & Carbendazim 12\% + Mancozeb 63\% (75 WP) & $2.5 \mathrm{~g}$ \\
\hline $\mathbf{T}_{\mathbf{5}}$ & Trichoderma harzianum $\left(5 \times 10^{7}\right.$ cfu/g carrier) & $10 \mathrm{~g}$ \\
\hline $\mathbf{T}_{\mathbf{6}}$ & Trichoderma viride $\left(5 \times 10^{7}\right.$ cfu/g carrier) & $10 \mathrm{~g}$ \\
\hline $\mathbf{T}_{\mathbf{7}}$ & Carbendazim 50 WP + T. harzianum + Neem seed cake & $1.0 \mathrm{~g}+10 \mathrm{~g}+5 \mathrm{q}$ \\
\hline $\mathbf{T}_{\mathbf{8}}$ & Carbendazim 50 WP + T. viride + Neem seed cake & $1.0 \mathrm{~g}+10 \mathrm{~g}+5 \mathrm{q}$ \\
\hline $\mathbf{T}_{\mathbf{9}}$ & $\begin{array}{l}\text { Carboxin 37.5\% + Thiram 37.5\% }(75 \mathrm{WP})+T . \\
\text { harzianum + Neem seed cake }\end{array}$ & $3.0 \mathrm{~g}+10 \mathrm{~g}+5 \mathrm{q}$ \\
\hline $\mathbf{T}_{\mathbf{1 0}}$ & $\begin{array}{l}\text { Carbendazim 12 \% + Mancozeb 63 \% (75 WP) + T. viride } \\
+ \text { Neem seed cake }\end{array}$ & $2.5 \mathrm{~g}+10 \mathrm{~g}+5 \mathrm{q}$ \\
\hline $\mathbf{T}_{\mathbf{1 1}}$ & Bioagents consortia & $10 \mathrm{~g}$ \\
\hline $\mathbf{T}_{\mathbf{1 2}}$ & Control & - \\
\hline
\end{tabular}


Table.2 Effect of integrated use of fungicide, bio-control agent and organic amendment on collar rot of chilli (Pooled)

\begin{tabular}{|c|c|c|c|c|c|c|c|c|c|}
\hline \multirow{2}{*}{$\begin{array}{l}\text { Tr. } \\
\text { No. }\end{array}$} & \multirow[t]{2}{*}{ Treatments } & \multirow{2}{*}{$\begin{array}{l}\text { Doses } \\
\text { (g/l of water or } \\
\text { q/ha of soil) }\end{array}$} & \multicolumn{3}{|c|}{ Per cent Incidence } & \multicolumn{3}{|c|}{ Yield (q/ha) } & \multirow{2}{*}{$\begin{array}{l}\text { Per cent increase } \\
\text { over control }\end{array}$} \\
\hline & & & 2016-17 & 2017-18 & Mean & 2016-17 & 2017-18 & Mean & \\
\hline $\mathbf{T}_{1}$ & Captan $50 \mathrm{WP}$ & $3.0 \mathrm{~g}$ & $\begin{array}{c}25.00 \\
(30.00)^{*}\end{array}$ & $\begin{array}{c}23.81 \\
(29.11)\end{array}$ & 24.41 & 110.60 & 104.10 & 107.35 & 41.38 \\
\hline $\mathbf{T}_{2}$ & Carbendazim $50 \mathrm{WP}$ & $1.0 \mathrm{~g}$ & $\begin{array}{l}23.81 \\
(29.19)\end{array}$ & $\begin{array}{c}21.43 \\
(27.58)\end{array}$ & 22.62 & 111.81 & 106.22 & 109.01 & 43.57 \\
\hline $\mathbf{T}_{3}$ & $\begin{array}{c}\text { Carboxin } 37.5 \%+\text { Thiram } 37.5 \% \\
\text { (75 WP) }\end{array}$ & $3.0 \mathrm{~g}$ & $\begin{array}{l}14.29 \\
(22.10)\end{array}$ & $\begin{array}{l}11.91 \\
(20.14)\end{array}$ & 13.10 & 121.03 & 115.21 & 118.12 & 55.56 \\
\hline $\mathbf{T}_{4}$ & $\begin{array}{c}\text { Carbendazim } 12 \%+\text { Mancozeb } 63 \\
\%(75 \mathrm{WP})\end{array}$ & $2.5 \mathrm{~g}$ & $\begin{array}{c}22.62 \\
(28.38)\end{array}$ & $\begin{array}{l}19.05 \\
(25.86)\end{array}$ & 20.84 & 115.00 & 108.73 & 111.87 & 47.33 \\
\hline $\mathbf{T}_{5}$ & $\begin{array}{l}\text { Trichoderma harzianum }\left(5 \times 10^{7}\right. \\
\text { cfu/g carrier) }\end{array}$ & $10 \mathrm{~g}$ & $\begin{array}{c}26.19 \\
(30.77)\end{array}$ & $\begin{array}{l}25.00 \\
(29.96)\end{array}$ & 25.60 & 100.40 & 94.31 & 97.35 & 28.21 \\
\hline $\mathbf{T}_{6}$ & $\begin{array}{l}\text { Trichodermaviride }\left(5 \times 10^{7} \mathrm{cfu} / \mathrm{g}\right. \\
\text { carrier })\end{array}$ & $10 \mathrm{~g}$ & $\begin{array}{l}27.38 \\
(31.54)\end{array}$ & $\begin{array}{c}28.57 \\
(32.31)\end{array}$ & 27.98 & 99.47 & 90.87 & 95.17 & 25.34 \\
\hline $\mathbf{T}_{7}$ & $\begin{array}{c}\text { Carbendazim } 50 \mathrm{WP}+T \text {. } \\
\text { harzianum+ Neem seed cake }\end{array}$ & $1.0 g+10 g+5 q$ & $\begin{array}{l}11.90 \\
(20.14)\end{array}$ & $\begin{array}{c}9.52 \\
(17.90)\end{array}$ & 10.71 & 129.63 & 123.28 & 126.46 & 66.55 \\
\hline $\mathbf{T}_{8}$ & $\begin{array}{c}\text { Carbendazim } 50 \mathrm{WP}+T \text {. viride+ } \\
\text { Neem seed cake }\end{array}$ & $1.0 g+10 g+5 q$ & $\begin{array}{l}13.10 \\
(21.18)\end{array}$ & $\begin{array}{c}10.71 \\
(19.10)\end{array}$ & 11.91 & 128.21 & 122.22 & 125.22 & 64.92 \\
\hline $\mathbf{T}_{9}$ & $\begin{array}{c}\text { Carboxin } 37.5 \%+\text { Thiram } 37.5 \% \\
(75 \mathrm{WP})+T . \text { harzianum + Neem } \\
\text { seed cake }\end{array}$ & $3.0 \mathrm{~g}+10 \mathrm{~g}+5 \mathrm{q}$ & $\begin{array}{c}4.76 \\
(17.90)\end{array}$ & $\begin{array}{c}3.57 \\
(15.50)\end{array}$ & 4.17 & 151.06 & 145.24 & 148.15 & 95.11 \\
\hline $\mathbf{T}_{10}$ & $\begin{array}{c}\text { Carbendazim } 12 \%+\text { Mancozeb } 63 \\
\%(75 \text { WP })+T . \text { viride }+ \text { Neem } \\
\text { seed cake }\end{array}$ & $2.5 g+10 g+5 q$ & $\begin{array}{c}9.52 \\
(12.43)\end{array}$ & $\begin{array}{c}7.14 \\
(10.89)\end{array}$ & 8.33 & 131.48 & 124.74 & 128.11 & 68.72 \\
\hline $\mathbf{T}_{11}$ & Bioagents consortia & $10 \mathrm{~g}$ & $\begin{array}{l}19.05 \\
(25.86)\end{array}$ & $\begin{array}{l}14.28 \\
(22.11)\end{array}$ & 16.67 & 119.00 & 112.70 & 115.85 & 52.57 \\
\hline $\mathbf{T}_{12}$ & Control & - & $\begin{array}{c}35.71 \\
(36.70)\end{array}$ & $\begin{array}{c}36.90 \\
(37.40)\end{array}$ & 36.31 & 79.37 & 72.49 & 75.93 & 0.00 \\
\hline & S.E.m \pm & & 1.03 & 0.89 & & 6.35 & 6.02 & 3.84 & - \\
\hline & C.D. $(P=0.05)$ & & 3.04 & 2.63 & & 18.78 & 17.75 & 11.11 & - \\
\hline
\end{tabular}

* Figures in parenthesis are angular transformed values 
Table.3 Economics of integrated management treatments on collar rot of chilli

\begin{tabular}{|c|c|c|c|c|c|c|c|c|}
\hline Tr. & Treatments & Yield & & & Gross & Net profit & \multicolumn{2}{|c|}{$\mathrm{B}: \mathrm{C}$ ratio } \\
\hline $\mathbf{T}_{1}$ & Captan $50 \mathrm{WP}$ & 107.35 & 700 & 85935 & 322051 & 236116 & 2.75 & $: 1$ \\
\hline $\mathbf{T}_{2}$ & Carbendazim $50 \mathrm{WP}$ & 109.02 & 920 & 86313 & 327040 & 240727 & 2.79 & $: 1$ \\
\hline $\mathbf{T}_{3}$ & Carboxin $37.5 \%$ + Thiram $37.5 \%$ (75 WP) & 118.12 & 1100 & 87358 & 354360 & 267002 & 3.06 & $: 1$ \\
\hline $\mathbf{T}_{4}$ & Carbendazim $12 \%$ + Mancozeb $63 \%$ (75 WP) & 111.87 & 560 & 86225 & 335610 & 249385 & 2.89 & $: 1$ \\
\hline $\mathbf{T}_{5}$ & Trichoderma harzianum $\left(5 \times 10^{7} \mathrm{cfu} / \mathrm{g}\right.$ carrier $)$ & 97.36 & 2000 & 86286 & 292063 & 205778 & 2.38 & $: 1$ \\
\hline $\mathbf{T}_{6}$ & Trichoderma viride $\left(5 \times 10^{7} \mathrm{cfu} / \mathrm{g}\right.$ carrier $)$ & 95.17 & 2000 & 86078 & 285516 & 199438 & 2.32 & $: 1$ \\
\hline $\mathbf{T}_{7}$ & $\begin{array}{c}\text { Carbendazim } 50 \mathrm{WP}+T . \text { harzianum }+ \text { Neem } \\
\text { seed cake }\end{array}$ & 126.46 & 5120 & 92170 & 379365 & 287195 & 3.12 & $: 1$ \\
\hline $\mathbf{T}_{8}$ & $\begin{array}{c}\text { Carbendazim } 50 \mathrm{WP}+T \text {. viride }+ \text { Neem seed } \\
\text { cake }\end{array}$ & 125.22 & 5120 & 92053 & 375648 & 283596 & 3.08 & $: 1$ \\
\hline $\mathbf{T}_{9}$ & $\begin{array}{c}\text { Carboxin } 37.5 \%+\text { Thiram } 37.5 \%(75 \mathrm{WP})+T \\
\text { harzianum + Neem seed cake }\end{array}$ & 148.15 & 5300 & 92507 & 384325 & 291818 & 3.15 & $: 1$ \\
\hline $\mathbf{T}_{10}$ & $\begin{array}{c}\text { Carbendazim } 12 \%+\text { Mancozeb } 63 \%(75 \mathrm{WP}) \\
+T . \text { viride }+ \text { Neem seed cake }\end{array}$ & 128.11 & 4760 & 93871 & 444444 & 350573 & 3.73 & $: 1$ \\
\hline $\mathbf{T}_{11}$ & Bioagents consortia & 115.85 & 2200 & 88243 & 347548 & 259305 & 2.94 & $: 1$ \\
\hline $\mathbf{T}_{12}$ & Control & 75.93 & 0 & 82250 & 227778 & 145528 & 1.77 & $: 1$ \\
\hline \multicolumn{9}{|c|}{ Average market price Rate Rs/q: 3000} \\
\hline
\end{tabular}


The results obtained in this study revealed that based on cost-benefit ratio chilli seedling dip treatment with Carbendazim $12 \%+$ Mancozeb 63 \% (75 WP) @ $2.5 \mathrm{~g} / \mathrm{l}+$ Trichoderma viride @ $10 \mathrm{~g} / \mathrm{l}+$ soil application with Neem seed cake @ 5 q/ha for fifteen minutes before transplanting is very effective in reducing the collar rot of chilli disease incidence, thereby increasing fruit yield. This may be an effective module for the integrated management of chilli collar rot.

\section{References}

Baker, K. F. (1987). Evolving concepts of biological control of plant pathogens. Annu. Rev. Phytopathol. 25: 67-85.

Banyal, D. K., Mankotia, V. and Sugha, S. K. (2008). Integrated management of tomato collar rot caused by Sclerotium rolfsii. J. Mycol. Pl. Pathol. 38 (2): 164167.

Begum, H. A., Akhtar, M. N. and Salam, M. A. (2011). Integrated management of collar rot of tomato. Int. J. Sustain. Crop Prod. 6 (2): 29-35.

Bhattacharjee, R., Suryawanshi, A. P. and Chavan, P. G. (2015). Integrated management of Sclerotium rolfsii, the incitants of root rot complex of chilli. Trends Biosci. 8 (1): 163-168.

Bhoraniya, M. F., Khandar, R. R., Khunti, J. P. (2003). Integrated Management of Stem rot of chilli with fungicides, oil cakes and biocontrol agent. Trichoderma harzianum. Adv. Plant Sci. 16 (1): 59-61.

Dutta, P. and Das, B. C. (2002). Management of collar rot of tomato by Trichoderma spp. and chemicals. Indian Phytopath. 55 (2): 235-237.

Gogoi, N. K., Phookan, A. K. and Narzary, B. D. (2004). Effect of Trichoderma harzianum and fungicides on plant growth and intensity of collar rot of elephant foot yam (Amorphophallus paeoniifolius). J. Root Crops. 30 (1): 76-79.

Jadon, K. S. (2009). Eco-friendly management of brinjal collar rot caused by Sclerotium rolfsii Sacc. Indian Phytopath. 62 (3): 345-347.

Kowsari, M., Motallebi, M. and Zamani, R.M. (2014). Construction of new GFPtagged fusants for Trichoderma harzianum with enhanced biocontrol activity. J. Plant Prot. Res.54, 122-131.

Kuldhar, D. P. and Suryawanshi A. P. (2017). Integrated management of stem rot and pod rot (Sclerotium rolfsii) of groundnut (Arachis hypogaea L.). Agric. Update, 12 (TECHSEAR-1): 238-246.

Lorito, M., Farkas, V., Rebuffat, S., Bodo, B. and Kubicek, C. P. (1996). Cell-wall synthesis is a major target of mycoparasitic antagonism by Trichoderma harzianum. J. Bacteriol. 178: 6382-6385.

Madhavi, B. G. and Bhattiprolu, S. L. (2011). Integrated disease management of dry root rot of chilli incited by Sclerotium rolfsii (Sacc.) Int. J. Pl. Ani. Environ. Sci. 1 (2): 31-37.

Madhuri, V. and Gayathri, D. A. (2014). Root rot of chilli incited by Sclerotium rolfsii Sacc. and its management. A review. Int. J. Appl. Bio. Pharm. Tech. 5: 197204.

Mahato, A., Mohan Kumar Biswas, M. K. and Suman Patra, S. (2017). Eco-friendly management of collar rot disease of tomato caused by Sclerotium rolfsii (Sacc.).Int. J. Pure App. Biosci. 5 (1): 513-520.

Mathur, K. and Gurjar, R. B. S. (2001). Sclerotium rolfsii- a new threat to chilli in Rajasthan. J. Mycol. Pl. Pathol. 31: 261.

Okereke, V. C., Wokocha, R.C. and Egein, M. I. G. (2007). Evaluation of Trichoderma harzianum, some botanicals and fungicides on Sclerotium 
wilt of potted tomato. Agric. J. 2 (5): 555-558.

Papavizas, G. C. (1985). Trichoderma and Gliocladium: Biology, ecology and potential for biocontrol. Ann. Rev. Phytopathol. 23: 23-54.

Punja, Z. K. (1985). The biology, ecology and control of Sclerotium rolfsii. Ann. Rev. Phytopathol. 23: 97-127.

Shafique, H.A., Sultana, V., EhteshamulHaque, S. and Athar, M. (2016). Management of soil-borne diseases of organic vegetables. J. Plant Prot. Res. , 56, 221-230.

Singh, D. and Dhancholia, S. (1991). A noteworthy disease of brinjal caused by Sclerotium rolfsii Sacc. Himachal Pradesh J. Agric. Res. 17 (1\&2): 119120.

Singh, U. M. A. and Thapliyal, P. N. (1998). Effect of inoculum density, host cultivars and seed treatment on the seed and seedling rot of soybean caused by
Sclerotium rolfsii. Indian Phytopath.51: 244-246.

Sultana, J. N., Pervez, Z., Rahman, H. and Islam, M. S. (2012). Integrated management for mitigating root rot of chilli caused by Sclerotium rolfsii. Bangladesh Res. Publ. J. 6 (3): 270-280.

Suryawanshi, A. P., Borgaonkar, A. S., Kuldhar, D. P. and Dey, U. (2015). Integrated management of collar rot (Sclerotium rolfsii) of brinjal (Solanum melongena). Indian Phytopath. 68 (2):189-195.

Vanitha, S. and Suresh, M. (2002). Management of collar rot of brinjal (Sclerotium rolfsii) by non-chemical methods. South Indian Hort. 50 (4-6): 602-606.

$\mathrm{Xu}, \mathrm{Y} . \mathrm{H}$. (2009). Occurrence and integrated management of chilli root rot caused by Sclerotium rolfsii. Plant Doctor. 22: 1920.

\section{How to cite this article:}

Daunde. A. T., K. T. Apet, K. D. Navgire and Khandare. V. S. 2020. Integrated Management of Collar Rot of Chilli Caused by Sclerotium rolfsii Sacc. Int.J.Curr.Microbiol.App.Sci. 9(06): 2187-2194. doi: https://doi.org/10.20546/ijcmas.2020.906.267 